\title{
Incremental diagnostic role of cardiac MRI in young-middle aged patients with high-grade atrio-ventricular block
}

\author{
Anna Baritussio, Amardeep Ghosh Dastidar, Nauman Ahmed, Jonathan Rodrigues, Antonio Frontera, \\ Chris B Lawton, Daniel Augustine, Elisa McAlindon, Chiara Bucciarelli-Ducci
}

From 19th Annual SCMR Scientific Sessions

Los Angeles, CA, USA. 27-30 January 2016

\section{Background}

Atrio-ventricular (AV) block is a common bradyarrhythmia in the elderly, but is a rare event in young or middle-aged adults, often leading to pacemaker implantation without further investigation, though underlying aetiology influences both treatment strategies and prognosis. Cardiovascular magnetic resonance (CMR) has the potential to identify an underlying aetiology for AV block, over and above transthoracic echocardiogram (TTE), which is offered as the first imaging technique. We sought to assess the diagnostic additive role of CMR in young and middle aged adults (18-60 years) with high-grade AV block and to determine which findings on CMR best predict clinical impact.

\section{Methods}

We retrospectively analysed the CMR registry from a tertiary centre in the South-West of England to collect data on consecutive high-grade AV block patients (1860 yrs) referred for CMR between September 2012 to July 2015. High-grade AVB was defined as the evidence of Mobitz II $2^{\text {nd }}$ degree or complete AVB on resting electrocardiogram (ECG). Patients underwent TTE and a comprehensive CMR protocol (including long and short axis cines, and late gadolinium enhancement, LGE, imaging). A change in diagnosis was defined as CMR findings leading to a new diagnosis compared to a multi-parametric pre-CMR diagnosis (clinical data, ECG and TTE).

\footnotetext{
Bristol NIHR Cardiovascular Biomedical Research Unit, Bristol Heart Institute,
} Bristol, UK
Multivariable associations of demographic and CMR characteristics with subsequent clinical impact. LVEF, left ventricular ejection fraction; LViEDV, left ventricular indexed end-diastolic volume; LViSV, left ventricular indexed stroke volume; RVEF, right ventricular ejection fraction; LGE, late gadolinium volume; RVEF,
enhancement.

\section{Results}

identified 31 patients with AV block (17 male, 55\%) ischemic heart disease was found in $3(10 \%)$ patients, heart in $15(48 \%)$. As compared to pre-CMR diagnosis, CMR findings led to a change in diagnosis in $45 \%$ of patients. In a multivariate model adjusting for demographic and CMR characteristics, only LGE was a signifindependent predictor of the underlying diagnosis

\section{Conclusions}

nagement of AVB in young is challenging. Our study

Table 1 Predictors of clinical impact

\begin{tabular}{ccccc}
\hline & Sig. & Exp (B) & \multicolumn{2}{c}{$\mathbf{9 5 \%}$ C.I. for EXP (B) } \\
\hline & & & Lower & Upper \\
\hline Gender & 0.184 & 4.226 & 0.505 & 35.383 \\
Age & 0.592 & 1.023 & 0.941 & 1.112 \\
LVEF & 0.081 & 1.230 & 0.975 & 1.552 \\
LViEDV & 0.253 & 1.019 & 0.986 & 1.053 \\
LViSV & 0.814 & 1.010 & 0.932 & 1.094 \\
RVEF & 0.209 & 0.847 & 0.654 & 1.098 \\
LGE & 0.042 & 8.174 & 1.075 & 62.139
\end{tabular}


independent predictor of a new diagnosis compared to other imaging characteristics like biventricular ejection fraction and volumes. CMR should be included in the diagnostic work up of young patients with high grade AVB.

Published: 27 January 2016

doi:10.1186/1532-429X-18-S1-0127

Cite this article as: Baritussio et al:: Incremental diagnostic role of cardiac MRI in young-middle aged patients with high-grade atrioventricular block. Journal of Cardiovascular Magnetic Resonance 2016 18(Suppl 1):0127.

Submit your next manuscript to BioMed Central and take full advantage of:

- Convenient online submission

- Thorough peer review

- No space constraints or color figure charges

- Immediate publication on acceptance

- Inclusion in PubMed, CAS, Scopus and Google Scholar

- Research which is freely available for redistribution

Submit your manuscript at www.biomedcentral.com/submit
Ciomed Central 